\title{
Sahlqvist Formulas Unleashed in Polyadic Modal Languages
}

\author{
VAlentin Goranko And Dimiter VAKarelov
}

\begin{abstract}
We propose a generalization of Sahlqvist formulas to polyadic modal languages by representing such languages in a combinatorial PDL style and thus, in particular, developing what we believe to be the right syntactic approach to Sahlqvist formulas at all. The class of polyadic Sahlqvist formulas PSF defined here expands essentially the so far known one. We prove first-order definability and canonicity for the class PSF.
\end{abstract}

\section{Introduction}

The quest for general frame-completeness results in modal logic has driven research ever since the first examples of incomplete modal logics were discovered by Thomason and Fine in the mid 1970's. One of the most general result of the sort was Sahlqvist theorem Sahlqvist 1975 where he proved two notable results for a large, syntactically defined class of modal formulas, called now Sahlqvist formulas: first, the correspondence result: they all define first-order conditions on Kripke frames and those conditions can be effectively "computed" from the modal formulas; and second, the completeness result: all these formulas are canonical, i.e. valid in their respective canonical frames, hence axiomatize completely the classes of frames determined by them. Some landmarks in the study of Sahlqvist formulas (SF) include, besides Sahlqvist's paper itself:

- Lemmon's conjecture in Lemmon and Scott 1977 on first-order definability and canonicity of a substantial subclass of Sahlqvist formulae, proven in Goldblatt 1976a, Goldblatt 1976b and independently confirmed and extended by Sahlqvist's result.

Advances in Modal Logic, Volume 3.

F. Wolter, H. Wansing, M. de Rijke, and M. Zakharyaschev, eds.

Copyright (C) 2002, CSLI Publications. 
- the systematic development in Benthem 1983 of Sahlqvist's algorithm for computing the first-order equivalent of a Sahlqvist formula, based on the substitution method;

- the modern approach to Sahlqvist formulas proposed in Sambin and Vaccaro 1989 , based on topological properties of descriptive frames allowing for unified treatment of first-order definability and canonicity.

- Kracht 1993 where the class of first-order formulas corresponding to Sahlqvist formulas was studied and a calculus computing the modal equivalent of such formulas is proposed, and further developed in Kracht 1999. (See also Blackburn et al. 2000 for a good exposition of Kracht's calculus.)

- Jónsson 1994 where an algebraic proof of canonicity of Sahlqvist formulas is given, building on ideas from Jónsson and Tarski 1952a. Actually, the latter can be regarded as a precursor and the earliest essential reference on the topic.

- Ghilardi and Meloni 1997, using new algebracic-topological ideas, prove an analogue of Sahlqvist's canonicity theorem for intermediate and intuitionistic modal logics.

Nice expositions of the ideas and technicalities around Sahlqvist's theorem can be found in Sambin and Vaccaro 1989, Gabbay et al. 1994, Blackburn et al. 2000, Chagrov and Zakharyaschev 1997.

Most of the results on Sahlqvist formulas are confined in the scope of monadic modal (or polymodal) logic. As de Rijke 1992 has shown, attempts for easy generalization for polyadic modal languages can fail badly. On the other hand, everyone who has worked on the topic must have felt that the phenomenon extends beyond the realm of the monadic languages, and actually, de Rijke and Venema in de Rijke 1993, Venema 1993, and de Rijke and Venema 1995, as well as Kracht and Wolter 1997 have proposed generalizations to the polyadic case. Blackburn et al. 2000 offers an insightful account of the first-order definability and canonicity results for the class of polyadic Sahlqvist formulas considered there.

All known to us approaches, however, follow the tradition of treating Sahlqvist formulas as implications ${ }^{1}$, where the consequent is a positive formula, and the antecedent satisfies some syntactic restrictions, so essentially the only avenue for extension of the class of Sahlqvist formulas has been to relax appropriately the restrictions on the antecedent. This may be the right way to go in the monadic case. The polyadic languages,

\footnotetext{
${ }^{1}$ A notable exception is Thm 9.10 from Benthem 1983 which defines a class of formulas syntactically larger, yet easily reducible to Sahlqvist formulas as defined here.
} 
however, offer a new perspective to the phenomenon, which can only be seen if the power of the polyadicity is fully unleashed, and this is what we are trying to demonstrate in this paper.

To start with, a well known observation which so far considered as trivial is that the disjunction and conjunction are actually dual to each other dyadic modal operators : $A \vee B=[=](A, B)$ and $A \wedge B=\langle=\rangle(A, B)$ with very simple Kripke semantics:

$$
M, x \models[=](A, B) \text { iff } \forall y_{1}, y_{2}\left(R^{=} x y_{1} y_{2} \rightarrow\left(M, y_{1} \models A \vee M, y_{2} \models B\right)\right) \text {, }
$$

where $R^{=} x y z$ holds iff $x=y=z$.

Using this observation one can eliminate all Boolean connectives, except for the negation, from a polyadic modal language, and replace them by respective modalities. This may seem just a gimmick, but in fact it offers a new way of dealing with (polyadic) modal formulas, and in particular with Sahlqvist formulas, as generalized modalities. ${ }^{2}$ Thus, one can work with a (almost) purely modal polyadic language defined in the style of PDL with test, where there is nothing else besides atoms and their negations, and polyadic diamonds and boxes. Here we use this style to develop a new treatment of Sahlqvist formulas, which, apart from extending their class, sheds a better light on their essence. In particular, this treatment allows us to give a more general definition of the class of Sahlqvist formulas in the polyadic case, which smoothly and naturally subsumes and even expands the monadic subclass, and also, to compactify the first-order definability and canonicity proofs.

The idea in a nutshell is that in purely modal polyadic languages, Sahlqvist formulas are represented by polyadic boxes the arguments of which are either positive formulas or formulas behaving as unary boxes over parametrically defined composite modal terms, which allow for firstorder definable minimal valuations satisfying them. For simple polyadic Sahlqvist formulas these valuations are computed at once, while the general case requires an inductive procedure. Furthermore, these minimal valuations are closed sets in an appropriate topology, which yields the canonicity.

The structure of the paper is as follows. The preliminary section 1 briefly introduces the largest class SF of Sahlqvist formulas in polyadic modal languages, known to us from the literature (see de Rijke and Venema 1995). In section 2 we introduce the purely modal polyadic languages,

\footnotetext{
${ }^{2}$ We have meanwhile found that the idea of composing boxes in polyadic languages has also been used in Givant and Venema 1999 to describe a class of equations preserved under completions of Boolean algebras with operators. This class is essentailly an algebraic translation of the class of polyadic Sahlqvist formulas defined in de Rijke and Venema 1995.
} 
define the class SPSF of simple polyadic Sahlqvist formulas then the full class of polyadic Sahlqvist formulas which in turn substantially extends SPSF. We show that the class of polyadic Sahlqvist formulas introduced here extends the so far known polyadic class both syntactically and semantically, and extends the monadic fragment at least syntactically. In section 3 we prove first-order definability of the polyadic Sahlqvist formulas. Section 4 is devoted to polyadic descriptive frames and contains the technical results needed to prove the canonicity of polyadic Sahlqvist formulas in section 5 .

\section{Sahlqvist formulas in classical polyadic languages.}

We assume basic familiarity with the syntax and semantics of the standard polyadic modal languages, a state-of-the-art reference for which is e.g. Blackburn et al. 2000, from where we quote some of the definitions below.

Consider an arbitrarily fixed polyadic modal language. Positive and negative formulas of the language are defined as usual.

Definition 1.1 Boxed atom is a formula $\mathbf{L}_{1} \ldots \mathbf{L}_{n} p$ where $\mathbf{L}_{1}, \ldots, \mathbf{L}_{n}$ is a (possibly empty) string of unary boxes and $p$ is a propositional variable.

Sahlqvist antecedent: a formula constructed from propositional constants, boxed atoms and negative formulas by applying $\vee, \wedge$, and diamonds of arbitrary arities.

Definite Sahlqvist antecedent is a Sahlqvist antecedent obtained without applying $\vee$ (i.e. constructed from propositional constants, boxed atoms and negative formulas by applying only $\wedge$ and diamonds of arbitrary arities).

(Definite) Sahlqvist implication: $A \rightarrow B$ where $A$ is a (definite) Sahlqvist antecedent and $B$ is a positive formula.

The following definition is combined from Blackburn et al. 2000 and de Rijke and Venema 1995.

Definition 1.2 (Definite) Sahlqvist formula ((D)SF): a formula constructed from (definite) Sahlqvist implications by freely applying unary boxes and conjunctions, and applying polyadic boxes and disjunctions to formulas sharing no common variables.

Basic Sahlqvist formula is a definite Sahlqvist formula obtained without applying conjunctions.

This class of polyadic Sahlqvist formulas will be denoted by dRV . The following easy observations will be used in the next section. 


\section{Proposition 1.3}

1. Every Sahlqvist implication is equivalent to a conjunction of definite Sahlqvist implications.

2. Every Sahlqvist formula from $d R V$ is equivalent to a conjunction of basic Sahlqvist formulas.

Remark 1.4 Kracht and Wolter 1997 too define a similar class of polyadic Sahlqvist formulas, using also definable operators like $(A, B)=$ $\square(A, B) \wedge \square(\neg A, B) \wedge \square(A, \neg B)$ which is actually equivalent to $\square(A, \perp) \wedge$ $\square(\perp, B)$, so that class does not extend dRV.

\section{Sahlqvist formulas in polyadic modal languages.}

\subsection{Purely modal polyadic languages.}

Definition 2.1 A purely modal polyadic language $\mathcal{L}_{\tau}$ contains propositional variables, negation $\neg$, and a modal similarity type $\tau$ consisting of a set of basic modal terms (modalities) with pre-assigned finite arities, including a 0 -ary modality $\iota_{0}$ a unary one $\iota_{1}$ and a binary one $\iota_{2}$.

The intuition behind the 3 distinguished modalities above is simple: $\iota_{0}$ will be interpreted as the constant $T$ and its dual as $\perp ; \iota_{1}$ will be the self-dual identity; $\iota_{2}$ will be $\vee$ and its dual $-\wedge$. Treating these connectives as modalities, besides allowing for elegance and uniformity, will provide suitable technical framework for working with Sahlqvist formulas.

Before we give the definition of a formula, let us stipulate that constant formula will mean a formula containing no variables.

Definition 2.2 By simultaneous mutual induction we define the set of modal terms $M T(\tau)$ and their arity function $\rho$, and the set of (purely) modal formulas $M F(\tau)$ as follows:

(MT ii) Every basic modal term is a modal term of the predefined arity.

(MT ii) Every constant formula is a 0-ary modal term.

(MT iii) If $n>0, \alpha, \beta_{1}, \ldots, \beta_{n} \in M T(\tau)$ and $\rho(\alpha)=n$, then $\alpha\left(\beta_{1}, \ldots, \beta_{n}\right) \in$ $M T(\tau)$ and $\rho\left(\alpha\left(\beta_{1}, \ldots, \beta_{n}\right)\right)=\rho\left(\beta_{1}\right)+\ldots+\rho\left(\beta_{n}\right)$.

Modal terms of arity 0 will be called modal constants.

(MF i) Every propositional variable is a modal formula.

(MF ii) Every modal constant is a modal formula.

(MF iii) If $A$ is a formula then $\neg A$ is a formula; 
(MF iv) If $A_{1}, \ldots, A_{n}$ are formulas, $\alpha$ is a modal term and $\rho(\alpha)=n>0$, then $[\alpha]\left(A_{1}, \ldots, A_{n}\right)$ is a modal formula.

Note that constant formulas and 0-ary terms are regarded as both modal terms and formulas. This ambiguity of the syntax should not cause confusion if properly handled, and we have put up with it for the sake of technical simplicity and convenience.

For technical purposes we extend the series of $\iota$ 's with n-ary modalities $\iota_{n}$ : inductively as follows: $\iota_{n+1}=\iota_{2}\left(\iota_{1}, \iota_{n}\right)$ for $n>1$. Furthermore, again for technical convenience, we can assume that the language contains transposers: operators $\theta_{i j}$ which swap the $i$-th and $j$-th argument of a modal term. We will not treat these transposers formally, but assuming them in the language will allow us not to be concerned with the specific ordering of the arguments in a modal formula.

Some notation on formulas:

$\langle\alpha\rangle\left(A_{1}, \ldots, A_{n}\right)=\neg[\alpha]\left(\neg A_{1}, \ldots, \neg A_{n}\right) ;$

$\mathrm{T}=\iota_{0}, \perp=\neg \iota_{0}$;

$A \vee B=\left[\iota_{2}\right](A, B), A \wedge B=\left\langle\iota_{2}\right\rangle(A, B)$, and respectively $A_{1} \vee \ldots \vee A_{n}=$ $\left[\iota_{n}\right]\left(A_{1}, \ldots, A_{n}\right), A_{1} \wedge \ldots \wedge A_{n}=\left\langle\iota_{n}\right\rangle\left(A_{1}, \ldots, A_{n}\right)$;

$A \rightarrow B=\neg A \vee B$.

Positive and negative occurrences of variables and positive and negative formulas are defined as usual.

One effect of the mutual definition of modal terms and formulas is that it allows construction of parametrized modal terms. For instance, if $\alpha$ is a unary term and $\beta$ is a binary one, then $\gamma=\beta\left(\neg[\beta]([\alpha](\perp), \top), \iota_{1}\right)$ is a unary modal term. Indeed, the formula $[\gamma] p$ can be essentially identified with $[\beta](\neg[\beta]([\alpha](\perp), \top), p)$, but the advantage of our approach is that this formula can now be treated just like a normal unary box-modality, which will be of important technical convenience.

The semantics of purely modal languages is a straightforward combination of the standard Kripke semantics for polyadic modal languages and PDL-type of polymodal languages, taking into account the fact that conjunctions and disjunctions are now treated as modalities. In particular, a $\tau$-frame is a structure $\left\langle W,\left\{R_{\alpha}\right\}_{\alpha \in M T(\tau)}\right\rangle$ where $R_{\alpha} \subseteq W^{\rho(\alpha)+1}$ is defined recursively by:

- $R_{\iota_{0}}=W, R_{\iota_{1}}=\{(x, x) \mid x \in W\}, R_{\iota_{2}}=\{(x, x, x) \mid x \in W\}$.

- $R_{\alpha\left(\beta_{1}, \ldots, \beta_{n}\right)}=\left\{\left(x, x_{11}, \ldots, x_{1 b_{1}}, \ldots, x_{n 1}, \ldots, x_{n b_{n}}\right) \subseteq W^{b_{1}+\ldots+b_{n}+1} \mid\right.$ $\left.\exists y_{1} \ldots y_{n}\left(R_{\alpha} x y_{1} \ldots y_{n} \wedge \bigwedge_{i=1}^{n} R_{\beta_{i}} y_{i} x_{i 1} \ldots x_{i b_{i}}\right)\right\}$ where $\rho\left(\beta_{i}\right)=b_{i}, i=1, \ldots n$.

Note that $R_{\iota_{n}}=\left\{(x, \ldots, x) \in W^{n+1} \mid x \in W\right\}$. 
Now, the truth definition of a formula at a state of a Kripke model extends the classical modal case with the clause (where $\bar{y}=y_{1} \ldots y_{n}$ ):

- $M, x \models[\alpha]\left(A_{1}, \ldots, A_{n}\right)$ iff $\forall \bar{y}\left(R_{\alpha} x y_{1} \ldots y_{n} \rightarrow \bigvee_{i=1}^{n} M, y_{i} \models A_{i}\right)$.

In particular, $M, x \models \alpha$ iff $R_{\alpha} x$ for any modal constant $\alpha$.

It follows immediately from the definitions that

$\left[\alpha\left(\beta_{1}, \ldots, \beta_{n}\right)\right]\left(A_{11}, \ldots, A_{1 n_{1}}, \ldots, A_{m 1}, \ldots, A_{m n_{m}}\right)$ is equivalent to $[\alpha]\left(\left[\beta_{1}\right]\left(A_{11}, \ldots, A_{1 n_{1}}\right), \ldots,\left[\beta_{n}\right]\left(A_{m 1}, \ldots, A_{m n_{m}}\right)\right)$.

Accordingly, the standard translation ST generalizes the one for monadic languages with the clauses:

- $S T(\sigma)=R_{\sigma}(x)$ for every modal constant $\sigma$;

- $S T\left([\alpha]\left(A_{1}, \ldots, A_{n}\right)=\forall \bar{y}\left(R_{\alpha} x y_{1} \ldots y_{n} \rightarrow \bigvee_{i=1}^{n} S T\left(A_{i}\right)\left(y_{i} / x\right)\right)\right.$

Again, note that all propositional logical connectives, as defined here, have their standard interpretation. Furthermore, the purely modal polyadic languages are equally expressive as the traditional ones.

\subsection{Simple Polyadic Sahlqvist formulas.}

Definition 2.3 Essentially positive formula (EPF) of a variable $p$ is a formula $A=[\alpha](p)$ or $A=[\alpha]\left(p, A_{1}, \ldots, A_{n}\right)$ where $A_{1}, \ldots, A_{n}$ are negative formulas not containing $p$. The variable $p$ in such a formula is called the essential variable of $A$, while all other variables are inessential in the formula.

Definition 2.4 A set of essentially positive formulas is: independent if no essential variable in a formula from the set occurs as an inessential variable in any formula from the set; separated if all EPFs have different essential variables; strongly independent if it is independent and separated.

Of course, the essential variable in an EPF need not be the first argument, but to simplify the notation we shall put it usually in first position.

Definition 2.5 Simple Polyadic Sahlqvist formula (SPSF) is any modal constant $\sigma$, or $A=[\alpha]\left(A_{1}, \ldots, A_{n}\right)$ where $\alpha$ is an $n$-ary modal term and each formula $A_{i}$ is either positive, or a negation of an essentially positive formula, and the subset of essentially positive formulas in $\left\{A_{1}, \ldots, A_{n}\right\}$ is independent.

Of course, we can close the class of SPSFs under conjunctions, but for technical reasons we will keep it as is. 


\section{$2.3 \quad$ Pre-processing}

Sahlqvist formulas are traditionally (here as well) defined syntactically, while the idea behind them is semantic. Thus, inessential syntactic manipulations, such as tautological transformations, can take a formula in or out of the class of these formulas without chainging its semantic properties. Here we briefly discuss how a purely modal polyadic formula can be pre-processed in a search for its representation as a SPSF, and how to present SPSFs in a canonical form. By $A \sim B$ we shall indicate that the formulas $A$ and $B$ are equivalent with respect to frame definability.

1. Tautological transformations can present the formula as a polyadic box (if necessary, by prefixing with $\left[\iota_{1}\right]$ ).

2. All monotone variables, that have only positive/negative occurrences in the formula, can be replaced resp. by $\perp / T$. Some easy logical equivalences can further simplify the formula.

3. Boxes can be pulled outwards and composed. Eventually the formula can be written as $[\alpha]\left(A_{1}, \ldots, A_{n}\right)$, where $A_{1}, \ldots, A_{n}$ are negated boxes or variables.

4. At this stage one can identify if the formula is a SPSF (or to a general PSF, to be introduced later) and if not, one can try to convert it into one, by an appropriate substitution, typically reversing the polarities of a variable $p$ by replacing it with $\neg p$. For example, $\left[\iota_{2}\right](\neg[\alpha](p, q),[\beta](p, q)) \sim\left[\iota_{2}\left(\iota_{1}, \beta\right)\right](\neg[\alpha](p, q), p, q) \sim$ $\left[\iota_{2}\left(\iota_{1}, \beta\right)\right](\neg[\alpha](\neg p, \neg q), \neg p, \neg q) \sim\left[\iota_{2}\left(\iota_{1}, \beta\right)\right](\langle\alpha\rangle(p, q), \neg p, \neg q)$.

5. Now, assuming the formula is a SPSF, every EPF $B$ can be regarded as a unary box over its essential variable, where all negative arguments of $B$ are regarded as parameters. Computing the relation corresponding to this composite box is straightforward from the definition.

6. Finally, an SPSF can be transformed to one, equivalent with respect to frame validity, with a strongly independent set of EPFs. This can be done by means of successive splitting a common essential variable of two EPFs into two different copies, illustrated by the following example:

$[\alpha]\left(\neg\left[\beta_{1}\right] p, \neg\left[\beta_{2}\right] p, P(p, \bar{q})\right) \sim[\alpha]\left(\neg\left[\beta_{1}\right] p_{1}, \neg\left[\beta_{2}\right] p_{2}, P\left(p_{1} \vee p_{2}, \bar{q}\right)\right)$, where $p_{1}, p_{2}$ are variables not occurring in $A$.

Actually, all these transformations can be easily performed deductively in the minimal polyadic modal logic.

Clearly, a series of such transformations can convert any SPSF into one with strongly independent set of EPFs, so every SPSF can be trans- 
formed into an -equivalent one in a canonical form:

$$
[\alpha]\left(\neg\left[\beta_{1}\right] p_{1}, \ldots \neg\left[\beta_{n}\right] p_{n}, C_{1}, \ldots C_{k}\right)
$$

where $\beta_{1}, \ldots \beta_{n}$ are unary modal terms, $p_{1}, \ldots, p_{n}$ are different propositional variables, and $C_{1}, \ldots C_{k}$ are positive formulas. In particular, each of $n$ and $k$ above can be 0 , and the canonical form may become a constant formula.

Hereafter, whenever suitable, we can assume that any SPSF has been pre-processed this way.

\subsection{SPSF subsumes dRV}

Lemma 2.6 Every definite Sahlqvist antecedent $A$ is equivalent to a negation of a SPSF in which all EPFs are boxed atoms.

Proof. Induction on $A$ :

- The cases of $A$ atomic, boxed atom or a negative formula are trivial, (note that every atomic formula is equivalent to a negation of a positive formula);

- $A=A_{1} \wedge A_{2}$,where $A_{1} \equiv \neg B_{1}$ and $A_{2} \equiv \neg B_{2}$ for some SPSFs $B_{1}$ and $B_{2}$. Then $A \equiv \neg\left[\iota_{2}\right]\left(B_{1}, B_{2}\right)$. Note that all EPFs in $\left[\iota_{2}\right]\left(B_{1}, B_{2}\right)$ are boxed atoms, hence still an independent set.

- $A=\langle\alpha\rangle\left(A_{1}, \ldots, A_{n}\right)$. Then $A \equiv \neg[\alpha]\left(\neg A_{1}, \ldots, \neg A_{n}\right)$ where each $\neg A_{1}$ is equivalent to a SPSF in which all EPFs are boxed atoms, hence so is $[\alpha]\left(\neg A_{1}, \ldots, \neg A_{n}\right)$.

$\dashv$

Proposition 2.7 Every formula from $d R V$ is equivalent to a conjunction of SPSFs.

Proof. Immediately from Lemmata 1.3 and 2.6. Note that if $A \rightarrow C$ is a SF and $A \equiv \neg B$ for some SPSF $B$ then $A \rightarrow C \equiv\left[\iota_{2}\right](B, C)$ is a SPSF, and also that applying disjunctions and polyadic boxes to SFs not sharing variables preserves the independence of the essential variables. $\dashv$

Actually, SPSF properly extends dRV. A simple example is

$$
[\mathbf{2}](\neg[\mathbf{2}](\perp, p),\langle\mathbf{2}\rangle(p, \top)),
$$

where $\mathbf{2}$ is a binary modality. It defines the frame condition $\forall x y z\left(R_{2} x y z \rightarrow \exists u v w\left(R_{2} y u v \wedge R_{2} z v w\right)\right)$.

Moreover, SPSF extend, at least syntactically the class of monadic SF as they allow the antecedent of a SF can take not only boxed atoms but any formulas of the type: $\square_{1}\left(A_{1} \rightarrow \square_{2}\left(A_{2} \rightarrow \ldots \square_{n}\left(A_{n} \rightarrow p\right) \ldots\right)\right.$ 
where $A_{1}, \ldots, A_{n}$ are positive formulas not containing $p$. Indeed, this is equivalent to $\square_{1}\left(\neg A_{1} \vee \square_{2}\left(\neg A_{2} \vee \ldots \square_{n}\left(\neg A_{n} \vee p\right) \ldots\right)\right.$ which can be written as an EPF $[\alpha]\left(\neg A_{1}, \neg A_{2}, \ldots, \neg A_{n}, p\right)$.

\subsection{Polyadic Sahlqvist formulas.}

Let $A=[\alpha]\left(\neg B_{1}, \ldots, \neg B_{n}, C_{1}, \ldots, C_{k}\right)$ where $B_{1}, \ldots, B_{n}$ are EPFs with essential variables resp. $p_{1}, \ldots, p_{n}$ which after pre-processing can be assumed different, and $C_{1}, \ldots, C_{k}$ are positive. In general, this need not have the virtues of a Sahlqvist formula. For instance, de Rijke 1992 has shown that $\left[\iota_{2}\right](\neg[\mathbf{2}]([\mathbf{2}](p, p), p),\langle\mathbf{2}\rangle(\langle\mathbf{2}\rangle(p, p), p))$ is not FO definable. A simpler example is $\left[\iota_{2}\right](\neg[\mathbf{2}](p, p),\langle\mathbf{2}\rangle(p, p))$ which defines the non-elementary frame condition "For every $x$ the binary relation $R_{x}$ on the remaining two variables $y$ and $z$ has an unoriented cycle of odd length."

Definition 2.8 Dependency digraph of the set of essential variables $\left\{p_{1}, \ldots, p_{n}\right\}$ of $A$ is a digraph $G=\left\langle\mathbf{V}_{A}, \mathbf{E}_{A}\right\rangle$ where $\mathbf{V}_{A}=\left\{p_{1}, \ldots, p_{n}\right\}$, and $p_{i} \mathbf{E}_{A} p_{j}$ iff $p_{i}$ occurs as an inessential variable in a formula from $B_{1}, \ldots, B_{n}$ with an essential variable $p_{j}$. A digraph is called acyclic if it does not contain oriented cycles.

Definition 2.9 A Polyadic Sahlqvist formula (PSF) is any modal constant $\sigma$, or $A=[\alpha]\left(A_{1}, \ldots, A_{n}\right)$ where $\alpha$ is an $n$-ary modal term and each formula $A_{i}$ is either positive, or a negation of an essentially positive formula, and the dependency digraph of the set of essential variables in $A$ is acyclic.

A variable in a PSF is essential if it has at least one occurrence as an essential variable in the formula, otherwise it is inessential.

The particular case when there are no arcs in the dependency digraph corresponds to the class of SPSFs.

The class Polyadic Sahlqvist formulas PSF can be considered closed under conjunctions.

Remark 2.10 PSFs can be assumed pre-processed, just like PSFSs, to a canonical form in which there are no inessential variables and every variable is essential in only one EPF. However, unlike SPSFs, not all EPFs can be made unary boxes.

Note that this pre-processing does not alter the (a)cyclicity of the dependency graph of the formula.

Example 2.11 The formula $[\mathbf{3}](\neg[\mathbf{1}] p, \neg[\mathbf{2}](\neg p, q),\langle\mathbf{1}\rangle[\mathbf{1}] q)$ is a PSF but not a simple one. 
PSF generalizes syntactically the classical monadic Sahlqvist formulas, but we do not know yet if it essentially extends their semantic conditions.

Example 2.12 Consider:

$$
D=p \wedge \square(\diamond p \rightarrow \square q) \rightarrow \diamond \square \square q .
$$

This is not a classical Sahlqvist formula and by now we do not know if it is semantically equivalent to one. In particular, its local FO correspondent:

$$
F O(D)=\exists y\left(R x y \wedge \forall z\left(R^{2} y z \rightarrow \exists u(R x u \wedge R u x \wedge R u z)\right)\right)
$$

is not a Kracht formula (see Kracht 1999 and Blackburn et al. 2000, Sect. 3.7). On the other hand, $D$ written in a purely modal polyadic language becomes a PSF:

$$
D=\left[\iota_{3}\right]\left(\neg p, \neg\left[\alpha\left(\iota_{2}(\alpha, \alpha)\right)\right](\neg p, q),\langle\alpha\rangle[\alpha] q\right),
$$

where $\alpha$ is the modal term corresponding to the $\square$.

However, this formula has a Sahlqvist equivalent in the classical tense language:

$$
D_{t}=p \rightarrow F G G P(F p \wedge P p) .
$$

\section{First-order definability of polyadic Sahlqvist formulas}

Theorem 3.1 Every polyadic Sahlqvist formula is first-order definable.

Proof. We adapt the Sahlqvist - van Benthem's algorithm. First we will demonstrate it on a simple PSF and then will extend it to arbitrary PSFs.

Let $A=[\alpha]\left(\neg B_{1}, \ldots, \neg B_{n}, C_{1}, \ldots, C_{k}\right)$ be a pre-processed PSF, where $\left\{B_{1}, \ldots, B_{n}\right\}$ is a separated set of EPFs and $C_{1}, \ldots, C_{k}$ are positive formulas. Let $\overline{\mathbf{q}}=q_{1}, \ldots, q_{n}$ be the variables occurring in $A, \overline{\mathbf{Q}}=Q_{1}, \ldots, Q_{n}$ be the respective unary predicate variables, and $\overline{\mathbf{y}}=y_{1}, \ldots, y_{n+k}$ be a string of fresh different individual variables. By $\overline{\mathrm{ST}(A)}$ we denote the secondorder closure of $\mathrm{ST}(A)$, which corresponds to frame validity. Then $\overline{\mathrm{ST}(A)}=$

$\forall x \forall \overline{\mathbf{Q}} \forall \overline{\mathbf{y}}\left(R_{\alpha} x y_{1} \ldots y_{n+k} \rightarrow\left(\bigvee_{i=1}^{n} \neg \mathrm{ST}\left(B_{i}\right)\left(y_{i} / x\right) \vee \bigvee_{i=1}^{k} \mathrm{ST}\left(C_{i}\right)\left(y_{n+i}\right)\right)\right.$ $\equiv \forall x \forall \overline{\mathbf{Q}} \forall \overline{\mathbf{y}}\left(R_{\alpha} x y_{1} \ldots y_{n+k} \wedge \bigwedge_{i=1}^{n} \operatorname{ST}\left(B_{i}\right)\left(y_{i} / x\right) \rightarrow \operatorname{POS}\left(y_{n+1}, \ldots, y_{n+k}\right)\right)$ for some positive formula $P O S$.

First, let us consider the particular case when $A$ is a SPSF, so all EPFs are unary boxes: $B_{j}=\left[\beta_{j}\right]\left(q_{j}\right)$ for some modal term $\beta_{j}$ and essential variable $q_{j}$. Then

$$
\mathrm{ST}\left(B_{j}\right)\left(y_{j} / x\right) \equiv \forall z_{j}\left(R_{\beta_{j}} y_{j} z_{j} \rightarrow Q_{j}\left(z_{j}\right)\right) .
$$


We now define the minimal valuation $V_{m}$ which makes these true:

$$
V_{m}\left(q_{j}\right)=R_{\beta_{j}}\left(y_{j}\right) .
$$

It is now easy to see that for any frame $\mathbf{F}$ and $x \in \mathbf{F}$ :

$$
\mathbf{F}, x \models \forall \overline{\mathbf{Q}} \mathrm{ST}(A) \text { iff } \mathbf{F}, x \models \operatorname{ST}(A)\left(\mathbf{V}_{m}(\overline{\mathbf{q}}) / \overline{\mathbf{Q}}\right),
$$

hence

$$
\mathbf{F}, x \models A \text { iff } \mathbf{F}, \mathbf{V}_{m}, x \models A .
$$

Indeed, $\overline{\mathrm{ST}(A)}=\forall x \forall \overline{\mathbf{y}} \forall \overline{\mathbf{Q}}(A N T(\overline{\mathbf{Q}}) \rightarrow \operatorname{POS}(\overline{\mathbf{Q}}))$ where $\overline{\mathbf{Q}}$ is the string of predicates corresponding to all essential variables and $A N T(\overline{\mathbf{Q}})=R_{\alpha} x y_{1} \ldots y_{n+k} \wedge \bigwedge_{i=1}^{n} \mathrm{ST}\left(B_{i}\right)\left(y_{i} / x\right)$.

Note that, once the parameters $x, y_{1}, \ldots, y_{n+k}$ are fixed consistently with $A N T$, the valuation $V_{m}$ is the minimal one (in set-theoretic sense) which makes each $B_{i}$, and hence $A N T(\overline{\mathbf{Q}})$, true. Therefore if $\mathbf{F}, \mathbf{V}_{m}, x \models$ $A$ then $\operatorname{POS}\left(V_{m}(\overline{\mathbf{q}})\right)$ is true. Now, take any valuation $V$. If it falsifies any $B_{i}$, then $A N T$ is rendered false, so the whole formula is true. Otherwise, $V_{m}(q) \subseteq V(q)$ for every essential variable $q$. Then, by monotonicity of positive formulas, $\operatorname{POS}\left(V_{m}(\overline{\mathbf{q}})\right) \rightarrow \operatorname{POS}(V(\overline{\mathbf{q}}))$ is valid, hence $\operatorname{POS}(V(\overline{\mathbf{q}}))$ is true, so the formula turns out true again.

Thus, $A$ defines the following first-order condition on frames:

$$
F O(A)=\forall x \forall \overline{\mathbf{y}}\left(R_{\alpha} x y_{1} \ldots y_{n+k} \rightarrow \operatorname{POS}\left(\mathbf{V}_{m}(\overline{\mathbf{q}}) / \overline{\mathbf{Q}}\right)\right) .
$$

Now, the proof for the general case of a PSF $A$ essentially repeats the one above. The key concern again is to define the right minimal valuation. Let $G_{A}$ be the dependency digraph of $A$. First, note that since $G_{A}$ does not contain cycles, it defines a strict partial ordering $\prec$ between the vertices: $q_{i} \prec q_{j}$ iff there is an arc path leading from $q_{i}$ to $q_{j}$. Following that partial ordering, a minimal valuation can be defined on the set of essential variables inductively as follows.

Suppose all $\prec$-predecessors (if any) of an essential variable $q$ have already been assigned values. Let the string of these predecessors be $\overline{\mathbf{q}}_{q}$ and let the string of second-order variables corresponding to them be $\overline{\mathbf{Q}}_{q}$.

Take any $\mathrm{EPF} B_{j}$ with an essential variable $q_{j}$. Then, as before:

$\operatorname{ST}\left(B_{j}\right)\left(y_{j} / x\right) \equiv$

$\forall z_{j} \forall \overline{\mathbf{u}_{j}}\left(R_{\beta_{j}} y_{j} z_{j} u_{j 1} \ldots u_{j n_{j}} \wedge \bigwedge_{i=1}^{n_{j}} \operatorname{ST}\left(P_{j i}\right)\left(u_{j i} / x\right) \rightarrow Q_{j}\left(z_{j}\right)\right)$.

Note that all variables occurring in any $\operatorname{ST}\left(P_{j i}\right)\left(u_{j i} / x\right)$ above correspond to predecessors of $q_{j}$, so they are amongst $\overline{\mathbf{Q}}_{q_{j}}$ and hence they have already been assigned their minimal values. 
Then we put

$\left.V_{m}\left(q_{j}\right)=\left\{z \mid \exists \overline{\mathbf{u}_{j}}\left(R_{\beta_{j}} y_{j} z u_{j 1} \ldots u_{j n_{j}} \wedge \bigwedge_{i=1}^{n_{j}} \operatorname{ST}\left(P_{j i}\right)\left(V_{m}\left(\overline{\mathbf{q}}_{j}\right) / \overline{\mathbf{Q}}_{q_{j}}\right)\left(u_{j i}\right)\right)\right)\right\}$.

In particular, if $\overline{\mathbf{q}}_{q_{j}}$ is empty, i.e. $q_{j}$ is $\prec$-minimal, then $V_{m}\left(q_{j}\right)$ is defined as before.

Now, an inductive argument on $\prec$ proves that $V_{m}$ has indeed the properties of the minimal valuation needed to prove first-order definability of $A$ as in the case of a SPSF.

Example 3.2 Let us compute the PSF formula from example 2.11: $B=[\mathbf{3}](\neg[\mathbf{1}] p, \neg[\mathbf{2}](\neg p, q),\langle\mathbf{1}\rangle[\mathbf{1}] q)$. Since $p \prec q$, we first compute $V_{m}(p)=R_{1}\left(y_{1}\right)$. Then $V_{m}(q)=\left\{z \mid \exists s\left(R_{2} y_{2} s z \wedge R_{1} y_{1} s\right)\right.$. Thus, $F O(B)=$ $\forall x y_{1} y_{2} y_{3}\left(R_{\mathbf{3}} x y_{1} y_{2} y_{3} \rightarrow \exists v\left(R_{1} y_{3} v \wedge \forall w\left(R_{\mathbf{1}} v w \rightarrow \exists s\left(R_{\mathbf{2}} y_{2} s w \wedge R_{1} y_{1} s\right)\right)\right)\right)$.

Remark 3.3 Note that in the latter example above, once $V_{m}(p)$ is determined, then $[\mathbf{2}](\neg p, q)$ can be regarded as a unary box $[\alpha](q)$ where $\alpha=\mathbf{2}\left(\neg V_{m}(p), \iota_{1}\right)$ is a unary parametrized modal term, the relation of which can be accordingly computed: $R_{\alpha} x y$ iff $\exists s\left(R_{2} x s y \wedge V_{m}(p)(s)\right)$. This trick will be essential in the proof of canonicity of PSFs.

\section{Polyadic descriptive frames}

In this section we obtain results about descriptive frames for polyadic modal languages which will be used further. Most of these will be generalizations of well-known properties of monadic descriptive frames, but we will establish some important relations between them and will present them in a way suitable for purely modal languages.

Let us fix an arbitrary purely modal language $\mathcal{L}(\tau)$.

Definition 4.1 Given a $\tau$-frame $F=\left\langle W,\left\{R_{\alpha}\right\}_{\alpha \in M T(\tau)}\right\rangle$, every n-ary modal term $\beta \in M T(\tau)$ defines an n-ary operator $\langle\beta\rangle$ on $\mathcal{P}(W)$ as follows:

$\langle\beta\rangle\left(X_{1}, \ldots, X_{n}\right)=\left\{x \in W \mid R_{\beta} x x_{1} \ldots x_{n}\right.$ for some $\left.x_{1} \in X_{1}, \ldots, x_{n} \in X_{n}\right\}$.

In particular, $\langle\beta\rangle=R_{\beta}$ for every 0 -ary term $\beta$.

Note that every operator $\langle\beta\rangle$ is monotone on each of its arguments.

Definition 4.2 A general frame for $\mathcal{L}(\tau)$ (general $\tau$-frame) is a structure $\left\langle W,\left\{R_{\alpha}\right\}_{\alpha \in M T(\tau)}, \mathbb{W}\right\rangle$ extending a $\tau$-frame with a Boolean algebra of admissible subsets of $\mathcal{P}(W)$, closed under the operators corresponding to the basic modal terms, and therefore under all operators $\langle\beta\rangle$. 
Every general $\tau$-frame $\mathfrak{F}=\left\langle W,\left\{R_{\alpha}\right\}_{\alpha \in M T(\tau)}, \mathbb{W}\right\rangle$ determines a topological space $T(\mathfrak{F})$ with a base of clopen sets $\mathbb{W} . T(\mathfrak{F})$ is a Hausdorff space with some additional properties which will be obtained further. For detailed study of this topology, its properties and usage in modal logic, see Sambin and Vaccaro 1988 and for topological treatment of Sahlqvist formulas see Sambin and Vaccaro 1989.

Hereafter, a closed set in a general $\tau$-frame will mean a subset of the domain closed with respect of the above mentioned topology, i.e. an intersection of a family of admissible sets.

We are going to extend the set of modal terms and respective class of operators to allow parametrization with closed sets.

Definition 4.3 Let $\mathfrak{F}=\left\langle W,\left\{R_{\alpha}\right\}_{\alpha \in M T(\tau)}, \mathbb{W}\right\rangle$ be a general $\tau$-frame. We define the set $\operatorname{PMT}(\tau, \mathfrak{F})$ of parametrized modal terms associated with $\mathfrak{F}$ and their respective operators on $\mathcal{P}(W)$ inductively as follows:

- $M T(\tau) \subseteq P M T(\tau, \mathfrak{F})$

- For every $(n+1)$-ary term $\beta \in P M T(\tau, \mathfrak{F})$ and a closed set $Z$ in $T(\mathfrak{F}), \beta(Z)$ is an $n$-ary term in $\operatorname{PMT}(\tau, \mathfrak{F})$ such that $\langle\beta(Z)\rangle\left(X_{1}, \ldots, X_{n}\right)=\langle\beta\rangle\left(X_{1}, \ldots, X_{n}, Z\right)$.

It is easy to see that $R_{\beta(Z)} x, x_{1}, \ldots, x_{n}$ iff there exists $x_{n+1} \in Z$ such that $R_{\beta} x x_{1} \ldots x_{n} x_{n+1}$.

Definition 4.4 Let $\mathfrak{F}=\left\langle W,\left\{R_{\alpha}\right\}_{\alpha \in M T(\tau)}, \mathbb{W}\right\rangle$ be a general $\tau$-frame and $\beta \in P M T(\tau, \mathfrak{F})$. The relation $R_{\beta}$ is tight in $\mathfrak{F}$ if the following condition holds: for any $x, x_{1}, \ldots, x_{n} \in W$,

$$
\begin{aligned}
& R_{\beta} x, x_{1}, \ldots, x_{n} \text { iff } \\
& \forall X_{1}, \ldots, X_{n} \in \mathbb{W}\left(x_{1} \in X_{1}, \ldots, x_{n} \in X_{n} \Rightarrow x \in\langle\beta\rangle\left(X_{1}, \ldots, X_{n}\right)\right) .
\end{aligned}
$$

Note that this condition is equivalent to: for every $x \in W$,

$x \in \bigcap\left\{\langle\beta\rangle\left(X_{1}, \ldots, X_{n}\right) \mid X_{1}, \ldots, X_{n} \in \mathbb{W} \quad \& \quad x_{1} \in X_{1}, \ldots, x_{n} \in X_{n}\right\}$.

In particular, every $R_{\beta}$ for a 0 -ary term $\beta$ is tight.

Definition 4.5 A family of sets $\mathcal{F}$ has the finite intersection property (FIP) if the intersection of every finite subfamily of $\mathcal{F}$ is nonempty.

Definition 4.6 A general $\tau$-frame $\left\langle W,\left\{R_{\alpha}\right\}_{\alpha \in M T(\tau)}, \mathbb{W}\right\rangle$ is:

- differentiated if for every $x, y \in W$, if $x \neq y$ then there is $X \in \mathbb{W}$ such that $x \in X$ and $y \notin \notin X$; 
- tight if for every basic modal term $\beta$ the relation $R_{\beta}$ is tight in $\mathfrak{F}$;

- compact if every family of admissible sets in $\mathfrak{F}$ with FIP has a non-empty intersection.

- refined if it is differentiated and tight.

- descriptive if it is refined and compact.

Note that:

- the property of being differentiated is expressed by the tightness of $R_{\iota_{1}}$, and so it becomes redundant. We keep it in the definition mainly to respect the tradition.

- compactness of a general $\tau$-frame $\mathfrak{F}$ as defined above is equivalent to the standard topological notion of compactness of $T(\mathfrak{F})$, i.e. every family of closed sets with the FIP has a non-empty intersection.

- by (a weaker version of) Tychonov's theorem, if $\mathfrak{F}$ is compact then for every $n \in N$, the product space $(T(\mathfrak{F}))^{n}$ is compact, too.

Hereafter, closedness of Cartesian products of sets will mean closedness in the respective product topology.

In the rest of this section we show that every descriptive $\tau$-frame has the properties that will be necessary to prove the canonicity of any PSF.

Lemma 4.7 In any differentiated $\tau$-frame $\mathfrak{F}=\left\langle W,\left\{R_{\alpha}\right\}_{\alpha \in M T(\tau)}, \mathbb{W}\right\rangle$, for any $n$-ary term $\beta \in P M T(\tau, \mathfrak{F}), R_{\beta}$ is tight iff for every $x \in W$ the set $R_{\beta}(x)=\left\{\left(x_{1}, \ldots, x_{n}\right) \mid R_{\beta} x x_{1} \ldots x_{n}\right\}$ is closed.

Proof. For 0-ary modal terms $\beta$ each of these conditions is trivially true, so we can assume that $\rho(\beta)>0$.

First, note that

$\forall X_{1}, \ldots, X_{n} \in \mathbb{W}\left(x_{1} \in X_{1}, \ldots, x_{n} \in X_{n} \Rightarrow x \in\langle\beta\rangle\left(X_{1}, \ldots, X_{n}\right)\right)$ iff $\forall X_{1}, \ldots, X_{n} \in \mathbb{W}\left(x \in[\beta]\left(-X_{1}, \ldots,-X_{n}\right) \Rightarrow\left(x_{1}, \ldots, x_{n}\right) \in-\left(X_{1} \times \ldots \times\right.\right.$ $\left.X_{n}\right)$ ).

Therefore, $R_{\beta}$ is tight iff for every $x \in W$, $R_{\beta}(x)=\bigcap\left\{-\left(X_{1} \times \ldots \times X_{n}\right) \mid X_{1}, \ldots, X_{n} \in \mathbb{W} \& x \in[\beta]\left(-X_{1}, \ldots,-X_{n}\right)\right\}$. $\dashv$

Definition 4.8 A family $\mathcal{F}$ of subsets of a set $X$ is called downwards directed if $\mathcal{F}$ contains a subset of the intersection of any two (and hence, any finitely many) members of $\mathcal{F}$.

Lemma 4.9 In any differentiated and compact general $\tau$-frame $\mathfrak{F}=$ $\left\langle W,\left\{R_{\alpha}\right\}_{\alpha \in M T(\tau)}, \mathbb{W}\right\rangle$, the following are equivalent for any $n$-ary term $\beta \in \operatorname{PMT}(\tau, \mathfrak{F}):$ 
(i) $R_{\beta}$ is tight.

(ii) (Esakia's lemma) For any downwards directed family $\left\{X_{1 i} \times \ldots \times\right.$ $\left.X_{n i}\right\}_{i \in I}$ of closed subsets of $W^{n}$,

$$
\bigcap_{i \in I}\left\{\langle\beta\rangle\left(X_{1 i}, \ldots, X_{n i}\right)\right\}=\langle\beta\rangle\left(\bigcap_{i \in I} X_{1 i}, \ldots, \bigcap_{i \in I} X_{n i}\right) .
$$

(iii) For every $x \in W$ the set $R_{\beta}(x)$ is closed.

Proof. Again, the non-trivial case is $\rho(\beta)>0$.

(i) $\Rightarrow$ (iii): Lemma 4.7 .

(iii) $\Rightarrow$ (ii): The inclusion $\supseteq$ follows from the monotonicity of $\langle\beta\rangle$.

For the converse inclusion, let $x \in \bigcap_{i \in I}\left\{\langle\beta\rangle\left(X_{1 i}, \ldots, X_{n i}\right)\right\}$. Then, due to the downwards directedness, the family of closed sets

$$
R_{\beta}(x) \cup\left\{X_{1 i} \times \ldots \times X_{n i}\right\}_{i \in I}
$$

has the FIP, so it has a non-empty intersection, i.e. there is a tuple $\left(x_{1}, \ldots, x_{n}\right)$ such that $R_{\beta} x x_{1} \ldots x_{n}$ and $\left(x_{1}, \ldots, x_{n}\right) \in \bigcap_{i \in I}\left\{\left(X_{1 i} \times \ldots \times\right.\right.$ $\left.X_{n i}\right\}=\bigcap_{i \in I} X_{1 i} \times \ldots \times \bigcap_{i \in I} X_{n i}$.

Therefore, $x \in\langle\beta\rangle\left(\bigcap_{i \in I} X_{1 i}, \ldots, \bigcap_{i \in I} X_{n i}\right)$.

(ii) $\Rightarrow$ (i):

The implication from left to right in the tightness condition for $R_{\beta}$ holds by definition. For the converse, it suffices to note that by (ii):

$\bigcap\left\{\langle\beta\rangle\left(X_{1}, \ldots, X_{n}\right) \mid X_{1}, \ldots, X_{n} \in \mathbb{W} \& x_{1} \in X_{1}, \ldots, x_{n} \in X_{n}\right\}=$ $\langle\beta\rangle\left(\left\{x_{1}\right\}, \ldots,\left\{x_{n}\right\}\right) . \quad \dashv$

Lemma 4.10 In every descriptive $\tau$-frame $\mathfrak{F}$, each of the conditions of Lemma 4.9 holds for every term $\beta \in P M T(\tau, \mathfrak{F})$.

Proof. We shall prove that Esakia's lemma holds for every $\beta$, by induction on $\beta$.

First, we will complete the induction on $M T(\tau)$.

For the basic terms tightness holds by definition, and hence the claim holds by Lemma 4.9 .

The inductive step for $\beta=\alpha\left(\alpha_{0}, \ldots, \alpha_{m}\right)$ is quite straightforward. Finally, suppose the claim holds for some $(n+1)$-ary term $\beta \in P M T(\tau, \mathfrak{F})$ and let $Z$ be a closed set in $T(\mathfrak{F})$. Then

$$
\begin{aligned}
& \langle\beta(Z)\rangle\left(\bigcap_{i \in I} X_{1 i}, \ldots, \bigcap_{i \in I} X_{n i}\right) \\
& =\langle\beta\rangle\left(\bigcap_{i \in I} X_{1 i}, \ldots, \bigcap_{i \in I} X_{n i}, \bigcap_{i \in I} Z\right) \\
& =\bigcap_{i \in I}\langle\beta\rangle\left(X_{1 i}, \ldots, X_{n i}, Z\right) \\
& =\bigcap_{i \in I}\langle\beta(Z)\rangle\left(X_{1 i}, \ldots, X_{n i}\right) . \quad \dashv
\end{aligned}
$$

Lemma 4.11 In any descriptive general $\tau$-frame $\mathfrak{F}$, for every positive formula $A\left(p_{1}, \ldots, p_{n}\right)$ the corresponding operator in $\mathfrak{F}$ 
$\lambda X_{1} \ldots X_{n} . A\left(X_{1}, \ldots, X_{n}\right)$ satisfies Esakia's lemma: for any downwards directed family of closed sets $\left\{X_{1 i} \times \ldots \times X_{n i}\right\}_{i \in I}$,

$$
\bigcap_{i \in I}\left\{A\left(X_{1 i}, \ldots, X_{n i}\right)\right\}=A\left(\bigcap_{i \in I} X_{1 i}, \ldots, \bigcap_{i \in I} X_{n i}\right) .
$$

In particular, for every positive formula $A\left(p_{1}, \ldots, p_{n}\right)$ the operator $\lambda X_{1} \ldots X_{n} . A\left(X_{1}, \ldots, X_{n}\right)$ is closed, i.e. $A\left(X_{1}, \ldots, X_{n}\right)$ is closed whenever $X_{1}, \ldots, X_{n}$ are closed.

Proof. First, note that every positive formula $A$ is equivalent to a formula built from propositional variables and modal constants applying only polyadic boxes and diamonds.

We shall prove the statement by induction on $A$, assuming it is constructed as above.

For propositional variables and modal constants the claim is trivial. For $\langle\beta\rangle\left(X_{1}, \ldots, X_{n}\right)$ the inductive step is the Esakia's lemma.

Finally, for $[\beta]\left(X_{1}, \ldots, X_{n}\right)$ the inductive step follows from the identity

$$
[\beta]\left(\bigcap_{i \in I} X_{1 i}, \ldots, \bigcap_{i \in I} X_{n i}\right)=\bigcap_{i_{1} \in I, \ldots, i_{n} \in I}[\beta]\left(X_{1 i_{1}}, \ldots, X_{n i_{n}}\right)
$$

which easily follows from the definition $[\beta]\left(X_{1}, \ldots, X_{n}\right)$, combined with the equality

$$
\bigcap_{i_{1} \in I, \ldots, i_{n} \in I}[\beta]\left(X_{1 i_{1}}, \ldots, X_{n i_{n}}\right)=\bigcap_{i \in I}[\beta]\left(X_{1 i}, \ldots, X_{n i}\right)
$$

which follows from the downward directedness. $\dashv$

\section{Canonicity of polyadic Sahlqvist formulas}

Theorem 5.1 Every polyadic Sahlqvist formula is canonical.

Proof. We show that every PSF is $d$-persistent following the scheme of the proof of canonicity of Sahlqvist formulas presented in Blackburn et al. 2000, to which the reader is referred for those technical details which would not differ in the general case presented here.

Let $A=[\alpha]\left(\neg B_{1}, \ldots, \neg B_{n}, C_{1}, \ldots, C_{k}\right)$ be a pre-processed PSF with EPFs $B_{1}, \ldots, B_{n}$ and different essential variables resp. $q_{1}, \ldots, q_{n}$. Let the dependency digraph of $A$ determine a partial order $\prec$ on these variables.

Let $\mathfrak{F}=\langle\mathbf{F}, \mathbb{W}\rangle$ be a descriptive general frame such that $\mathfrak{F} \models A$.

As we showed in the proof of theorem 3.1, if $\mathbf{F}, V_{m} \models A$ for the minimal valuation $V_{m}$, defined as before, then $\mathbf{F}, V \models A$ for any valuation $V$, so it suffices to prove that $\mathbf{F}, V_{m} \models A$. The problem is that the minimal valuation need not be admissible in $\mathfrak{F}$. However, it will suffice to show the following: 
(C1) $V_{m}$ is closed i.e. an intersection of admissible valuations.

(C2) For every closed valuation $U$ in $\mathfrak{F}$ and a positive formula $P$, $U(P)=\bigcap_{U \preceq V} V(P)$ where the intersection ranges over all admissible valuations $V$ which extend $U$.

For $(\mathrm{C} 1)$, we can restrict our consideration to the variables occurring in $A$, i.e. the essential variables $q_{1}, \ldots, q_{n}$. We shall prove by $\prec$-induction that every valuation

$$
V_{m}\left(q_{j}\right)=\left\{z \mid \exists \overline{\mathbf{u}_{j}}\left(R_{\beta_{j}} y_{j} z u_{j 1} \ldots u_{j n_{j}} \wedge \bigwedge_{i=1}^{n_{j}} \operatorname{ST}\left(P_{j i}\right)\left(V_{m}\left(\overline{\mathbf{q}}_{q_{j}}\right) / \overline{\mathbf{Q}}_{q_{j}}\right)\left(u_{j i}\right)\right)\right\}
$$

is of the type $R_{\beta}\left(y_{j}\right)$ for some $\beta \in P M T(\tau, \mathfrak{F})$, and hence, by Lemma 4.10 , is closed in $\mathfrak{F}$.

For the $\prec$-minimal variables the claim is immediate, because their respective EPFs are unary boxes.

Now suppose the claim holds for all predecessors $\overline{\mathbf{q}}_{p}$ of the variable $p=q_{j}$, i.e. for every $q_{i} \in \overline{\mathbf{q}}_{p}, V_{m}\left(q_{i}\right)=R_{\beta_{q_{i}}}$ for some $\beta_{q_{i}} \in \operatorname{PMT}(\tau, \mathfrak{F})$, and hence is closed.

Let $n_{j}=n$ and denote $C_{i}=P_{j i}\left(V_{m}\left(\overline{\mathbf{q}}_{p}\right)\right)$ for $i=1, \ldots, n$. Note that each $C_{i}$ is closed by the inductive hypothesis and Lemma 4.11, since $P_{j i}$ is positive.

Consider the unary term $\gamma=\beta\left(C_{n}\right) \ldots\left(C_{2}\right)\left(C_{1}\right) \in P M T(\tau, \mathfrak{F})$, i.e. such that $[\gamma](A)=[\beta]\left(A, C_{1}, \ldots, C_{n}\right)$. Then for any $z \in W, R_{\gamma} y z$ holds iff there exist $u_{1}, \ldots, u_{n}$ such that $R_{\beta} y z u_{1} \ldots u_{n}$ and $u_{i} \in C_{i}$ for $i=1, \ldots, n$. Therefore $V_{m}\left(p_{j}\right)=R_{\gamma}\left(y_{j}\right)$.

(C2) follows from Lemma 4.11.

Now, to complete the proof, let us see why $\mathbf{F}, V_{m} \models A$. As in the proof of theorem 3.1, let $\overline{\mathrm{ST}(A)}=\forall x \forall \overline{\mathbf{y}} \forall \overline{\mathbf{Q}}(A N T(\overline{\mathbf{Q}}) \rightarrow \operatorname{POS}(\overline{\mathbf{Q}}))$. Fix the parameters $x, \bar{y}$ consistently with $A N T$ (otherwise the formula turns vacuously true) and take any admissible valuation $U$ defined inductively on $\prec$ and extending $V_{m}$. It will render $A N T$ true, hence $P O S$ true, because $\mathfrak{F} \models A$. Then, by $(\mathrm{C} 2)$, POS will be true for $V_{m}$. $\dashv$

Remark 5.2 The conditions (C1) and (C2) in the proof above hold trivially for Kripke frames (i.e. full general frames), which allows for simultaneous treatment of first-order definability and canonicity of PSFs, in the spirit of Sambin and Vaccaro 1989 and Kracht 1993.

\section{Concluding remarks}

This work is certainly not the last word on Sahlqvist formulas. On the contrary, we believe that the approach developed here will allow for further generalizations and insights on them. An ultimate goal in this 
direction would be an explicit semantic characterization of the class of all formulas for which the proof of Sahlqvist's theorem work, through a deeper analysis of their semantic and topological nature.

An important question is to what extent polyadic Sahlqvist formulas can successfully co-operate in axiomatizations with non-orthodox rules of inference, a line of research initiated in Venema 1993. In a separate paper we study polyadic extensions of the class of Sahlqvist formulas in extended languages and additional rules.

Last but not least, proof systems for modal logic are still to catch up with the semantic richness and harmony of Sahlqvist formulas and make them equally useful in a deductive framework. In particular, "displaying" Sahlqvist formulas is a challenge to proof theorists.

\section{Acknowledgements}

We thank Johan van Benthem, Marcus Kracht, Silvio Ghilardi, and Yde Venema for useful discussions and comments. The work of both authors was partly supported by a research grant GUN 2034353 of the National Research Foundation of South Africa. The work of Valentin Goranko was partly done during his sabbatical leave, while visiting the University of Victoria, BC, Canada and supported in part by NSERC. He would like to thank Bruce Kapron who invited him for that visit.

\section{References}

Benthem, J. van. 1983. Modal Logic and Classical Logic. Naples: Bibliopolis.

Blackburn, P., M. de Rijke, and Y. Venema. 2000. Modal Logic. Cambridge Tracts in Theoretical Computer Science.

Chagrov, A., and M. Zakharyaschev. 1997. Modal Logic. Oxford Logic Guides, Vol. 35. Oxford: Oxford University Press.

de Rijke, M. 1992. How not to generalize Sahlqvist's Theorem. technical note. de Rijke, M. 1993. Extending Modal Logic. Doctoral dissertation, ILLC, University of Amsterdam.

de Rijke, M., and Y. Venema. 1995. Sahlqvist's Theorem for Boolean Algebras with Operators with an Application to Cylindric Algebras. Studia Logica 54:61-78.

Gabbay, D.M., I. Hodkinson, and M. Reynolds. 1994. Temporal Logic: Mathematical Foundations and Computational Aspects. Oxford University Press.

Ghilardi, S., and G. Meloni. 1997. Constructive Canonicity in Non-classical Logics. Annals of Pure and Applied Logic 86:1-32.

Givant, S., and Y. Venema. 1999. The Preservation of Sahlqvist's equations of Boolean Algebras with operators. Algebra Universalis 41:47-84.

Goldblatt, R.I. 1976a. Metamathematics of Modal Logic I. Reports on Mathematical Logic 6:41-78. 
Goldblatt, R.I. 1976b. Metamathematics of Modal Logic II. Reports on Mathematical Logic 7:21-52.

Jónsson, B. 1994. On the canonicity of Sahlqvist identities. Studia Logica $53: 473-491$.

Jónsson, B., and A. Tarski. 1952a. Boolean Algebras with Operators, Part I. American Journal of Mathematics 73:891-939.

Jónsson, J., and A. Tarski. 1952b. Boolean Algebras with Operators, Part II. American Journal of Mathematics 74:127-162.

Kanger, S. (ed.). 1975. Proceedings of the Third Scandinavian Logic Symposium. Uppsala 1973. Amsterdam. North-Holland.

Kracht, M. 1993. How Completeness and Correspondence Theory Got Married. In Diamonds and Defaults, ed. M. de Rijke, 175-214. Kluwer, Dordrecht.

Kracht, M. 1999. Tools and Techniques in Modal Logic. Elsevier.

Kracht, M., and F. Wolter. 1997. Simulation and Transfer Results in Modal Logic - A Survey. Studia Logica 59:149-177.

Lemmon, E.J., and D.S. Scott. 1977. The "Lemmon Notes": An Introduction to Modal Logic. Oxford: Blackwell.

Sahlqvist, H.1975. Completeness and Correspondence in the First and Second Order Semantics for Modal Logic. In Kanger Kanger 1975, 110-143.

Sambin, G., and V. Vaccaro. 1988. Topology and Duality in Modal Logic. Annals of Pure and Applied Logic 37:249-296.

Sambin, G., and V. Vaccaro. 1989. A New Proof of Sahlqvist's Theorem on Modal Definability and Completeness. Journal of Symbolic Logic 54:992999.

Venema, Y. 1993. Derivation rules as anti-axioms in modal logic. Journal of Symbolic Logic 58:1003-1034.

Valentin Goranko

Department of Mathematics, Rand Afrikaans University

PO Box 524, Auckland Park 2006, Johannesburg, South Africa

E-mail: vfg@na.rau.ac.za

URL: http://general.rau.ac.za/maths/goranko/

Dimiter Vakarelov

Department of Mathematical Logic with Laboratory for Applied Logic Faculty of Mathematics and Computer Science, Sofia University

blvd James Bouchier 5, 1126 Sofia, Bulgaria

E-mail: dvak@fmi.uni-sofia.bg 